\title{
A Search for Line Shape and Depth Variations in 51 Pegasi and $\tau$ Boötis
}

Timothy M. Brown, Rubina Kotak

High Altitude Observatory/National Center for Atmospheric Research * 3450 Mitchell Ln, P.O Box 3000, Boulder, CO 80307 brown@hao.ucar.edu, rubina@astro.lu.se

Scott D. Horner

Department of Astronomy \& Astrophysics, Pennsylvania State University

University Park, PA 16802

horner@astro.psu.edu

and

Edward J. Kennelly, Sylvain Korzennik, P. Nisenson \& Robert W. Noyes

Harvard-Smithsonian Center for Astrophysics, Cambridge MA 02138

tkennelly@cfa0.harvard.edu, sylvain@cfa0.harvard.edu, nisenson@cfa0.harvard.edu noyes@cfa0.harvard.edu

Received _-____-_-

* The National Center for Atmospheric Research is Sponsored by the National Science Foundation 


\begin{abstract}
Spectroscopic observations of 51 Pegasi and $\tau$ Boötis show no periodic changes in the shapes of their line profiles; these results for 51 Peg are in significant conflict with those reported by Gray and Hatzes (1997). Our detection limits are small enough to rule out nonradial pulsations as the cause of the variability in $\tau$ Boo, but not in $51 \mathrm{Peg}$. The absence of line shape changes is consistent with these stars' radial velocity variability arising from planetary-mass companions.
\end{abstract}

Subject headings: line: profiles — stars: individual (51 Peg, $\tau$ Boo) — stars: oscillations — stars: planetary systems

\title{
I. Introduction
}

Short-period radial velocity variability of several tens of $\mathrm{m} \mathrm{s}^{-1}$ has recently been detected in several Sun-like stars, suggesting the presence of planets with roughly jovian mass in orbits smaller than 0.25 AU (Mayor \& Queloz 1995, Butler et al 1997, Noyes et al. 1997). Gray (1997) (henceforth G97) and Gray \& Hatzes (1997) (henceforth GH) challenged this planetary interpretation, based on evidence for changes, synchronous with the radial velocity variation, in the spectrum of 51 Pegasi. The parameters monitored by G97 and GH were the shape of the $\lambda 6253$ Fe I line (parameterized by its bisector shape), and the ratio of the depths of $\lambda 6252 \mathrm{~V} \mathrm{I}$ and $\lambda 6253 \mathrm{Fe}$ I. Line profile changes would not be expected from the gravitational attraction of an orbiting body, but would be a natural consequence of dynamic processes (perhaps nonradial pulsations) in the stellar atmosphere. It is important to know whether Gray's challenge is correct; if it is, great efforts are being expended to explain planets that may not exist. We report here the results of an independent spectroscopic study of the stars 51 Peg and $\tau$ Boo, searching for confirming evidence for periodic variations in line shape or depth. In order to get the most information out of our moderate-resolution data we have introduced some new analysis techniques. These and the related modeling of line profiles that have been distorted by nonradial pulsations are described in Brown et al. (1998); here we shall report only our results.

\section{Observations and Modeling}

We obtained the observations described here using the Advanced Fiber Optic Echelle (AFOE) spectrograph at the $1.5 \mathrm{~m}$ Tillinghast telescope at Mt. Hopkins, AZ. This spectrograph is designed for precise radial velocity measurements, and employs several techniques to assure a stable spectrograph point spread function (Brown et al. 1994). We observed 51 Peg and $\tau$ Boo with the AFOE configured to give $\lambda / \delta \lambda=R \simeq 50000$. A typical observation of each star consisted of a sequence of 3 sequential integrations, each lasting $10 \mathrm{~m}$, and each giving $\mathrm{S} / \mathrm{N}$ of about 150 in the continuum near $600 \mathrm{~nm}$. Because they were intended for radial velocity studies, a large majority of our observations were taken using an $I_{2}$ absorption cell. In these data, the stellar spectrum between $500 \mathrm{~nm}$ and $610 \mathrm{~nm}$ is heavily blended with the $I_{2}$ spectrum, therefore we did not use this wavelength range in any of our line shape analysis. In most cases we took only one 3-integration sequence each night, but for a few nights we took 2 such sequences, one at the beginning of the night and one at the end. The observations of 51 Peg consist of 59 spectra, encompassing 
20 sequences taken on 18 separate nights between 1995 Nov. 2 and 1996 Jul. 26. Those of $\tau$ Boo consist of 90 spectra, 30 sequences, and 23 nights between 1996 Jun. 25 and 1997 Mar. 26. The $56 \mathrm{~m} \mathrm{~s}^{-1}$ radial velocity variation of $51 \mathrm{Peg}$ (period $4.231 \mathrm{~d}$ ) is clearly evident in these data, as is the much larger $\left(468 \mathrm{~m} \mathrm{~s}^{-1}, 3.313 \mathrm{~d}\right.$ ) variation of $\tau$ Boo.

The AFOE's spectral resolution is not high enough to allow useful measurements of spectrum line bisectors. We therefore adopted a different method for characterizing the shapes of spectrum lines - a decomposition in terms of Hermite functions $\mathcal{H}_{i}(\lambda / \sigma)$, defined by

$$
\mathcal{H}_{n}\left(\frac{\lambda}{\sigma}\right)=\frac{N_{n}}{\sqrt{\sigma}} \exp \left(-\frac{\lambda^{2}}{2 \sigma^{2}}\right) H_{n}\left(\frac{\lambda}{\sigma}\right)
$$

where $H_{n}(\lambda / \sigma)$ is a Hermite polynomial as defined by Abromowitz and Stegun (1972), and $N_{n}$ is a normalization factor given by $N_{n}=\left(2^{n} n ! \sqrt{\pi}\right)^{-1 / 2}$. The decomposition involved representing pieces of spectrum $I(\lambda)$ typically $1.5 \mathrm{~nm}$ wide and centered on a fiducial wavelength $\lambda_{f}$ as follows:

$$
I(\lambda)=C\left[1+S\left(\lambda-\lambda_{f}\right)\right]\left[1-\sum_{j} D_{j}\left(\mathcal{H}_{0}\left(\frac{\lambda-\lambda_{c j}}{\sigma}\right)+\sum_{i=3}^{5} h_{i} \mathcal{H}_{i}\left(\frac{\lambda-\lambda_{c j}}{\sigma}\right)\right)\right]
$$

where $C$ and $S$ describe the brightness and slope of the local continuum, $D_{j}$ is the depth relative to continuum brightness of the $j^{\text {th }}$ spectrum line, $\lambda_{c j}$ is the center wavelength of the $j^{\text {th }}$ line, and $\sigma$ and the coefficients $h_{i}$ describe the width and the departure from a pure Gaussian shape, assumed common to all the lines. The parts of the line profile described by $h_{1}$ and $h_{2}$ have been absorbed into $\lambda_{c}$ and $\sigma$, respectively. Thus, a time series of the $h_{i}$ coefficients contains all of the line shape information in our observations, while the ratio of the $D$ parameters for the $\lambda 6252 \mathrm{~V}$ I and the $\lambda 6253 \mathrm{Fe}$ I lines reproduces G97's measurement of line depth ratio. In what follows, we express the line shape coefficients $h_{i}$ as a percentage of $D_{j}$. For the analysis described below, we fit 3 (for $51 \mathrm{Peg}$ ) or 4 (for $\tau$ Boo) sections of spectrum, each about $1.5 \mathrm{~nm}$ wide. These wavelength ranges contained about 60 distinct spectrum lines for each star. This use of multiple lines to gain noise immunity is justified when seeking evidence for pulsations, since both theory and observation (of, e.g., $\delta$ Scuti stars - see Kennelly et al. 1997) indicate that pulsations modify line shapes in the same way for all but very strong spectral lines.

To compare our results with those of G97 and GH, we were obliged to model the line shapes emerging from pulsating and rotating stars, computing both Hermite expansion coefficients $h_{i}$ and line bisector shape indices for a wide range of nonradial pulsation parameters. The modeling process was similar to that described by, for example, Vogt \& Penrod (1983), by Hatzes (1996), and by Schrijvers et al. (1997). At each point on a fine grid sampling the observable stellar hemisphere, we computed the line-of-sight component of velocity resulting from the sum of rotation and pulsation. The line profiles emerging from each point were then added together with appropriate Doppler shifts and limb darkening to make up the observed profile from the complete star. We then convolved the diskintegrated profiles with instrumental point spread functions to give those that would have been observed with $R=100000$ (to simulate G97's data) and with $R=50000$ (to simulate 
the AFOE). Last, we analyzed a series of such line profiles computed at various phases of the oscillation cycle as if they were real data, to determine the relationship between measures of bisector shape and our own $h_{i}$ coefficients.

The principal conclusion from the modeling applies to slowly-rotating stars such as $51 \mathrm{Peg}$ and to fluid motions (such as those caused by pulsation) that are sensibly constant over the range of depths where the spectrum line is formed. In this case, our $h_{3}$ coefficient, the bisector span $S_{b}$, and bisector curvature $C_{b}$ all measure essentially the same quantity: the lowest-order antisymmetric distortion of the line profile that is not a simple line shift.

For the residual flux values used by $\mathrm{GH}$ in their line bisector studies $(.85, .71, .48)$ and a central line depth of 0.6 (appropriate for $\lambda 6253 \mathrm{Fe}$ I), the proportionality between $h_{3}, S_{b}$, and $C_{b}$ turns out to be

$$
\sigma h_{3} \simeq 0.35 S_{b} \simeq-2.2 C_{b}
$$

where $\sigma$ is the Gaussian line width parameter in velocity units. For processes (e.g. granulation) involving depth-dependent correlations between velocity and temperature, this equivalence need not hold. But for the circumstances that most concern us, $h_{3}$ is a good proxy for the bisector-based measures of line shape oscillation. We also computed the ratio between $h_{3}$ and the RV amplitude. This ratio is quite variable, depending upon the mode spherical harmonic degree $\ell$, the azimuthal order, the ratio of horizontal to vertical velocities, and the inclination of the pulsation axis. It is nevertheless possible to characterize the typical behavior for each $\ell$, which allowed us to estimate how large an $h_{3}$ variation to expect, given a measured radial velocity variation. Finally, we found that slowly rotating stars display little change in line shape for a given radial velocity signal, but that the shape changes grow more pronounced as $v \sin i$ starts to exceed the intrinsic line width. Some of these conclusions disagree in important ways with the results of similar modeling by GH; for more details, see Brown et al. (1998).

\section{Results of Time-Series Analysis}

\section{A. 51 Pegasi}

We searched for periodic signals in the $51 \mathrm{Peg}$ time series of $h_{i}$ and line depth ratio in two ways: we computed periodograms of the time series, and we fit the amplitudes and phases of sinusoidal functions with periods of $4.231 \mathrm{~d}$ (the radial velocity period) and $2.575 \mathrm{~d}$ (the period of the largest bisector curvature signal seen by GH). No obvious narrow-band signals appear in the periodograms at any frequency, for any of the $h_{i}$ or for the line strength ratio. We therefore used the periodograms merely to provide estimates of the noise in each of the parameters, using for this purpose the values within a frequency range of \pm 1 cycle per month about the periods of interest. Table 1 shows the results of the sinusoid fitting, with error estimates taken from the periodograms as just described. For $51 \mathrm{Peg}$, only the $h_{5}$ amplitudes and the line ratio amplitude for the $2.575 \mathrm{~d}$ period exceed zero by as much as the estimated error $\sigma$; the largest difference from zero is $1.7 \sigma$. For the $4.231 \mathrm{~d}$ radial velocity period, we measure the variation in line depth ratio to be a little less than half that reported by G97, but the uncertainty is such that our result is about equally consistent with G97's value or with no variation at all.

What does the non-detection of an $h_{3}$ signal mean in physical terms? Two kinds of 
answer are possible, as illustrated in Figure 1. The line bisector curvature and the $h_{3}$ fitting coefficient measure similar characteristics of spectral line asymmetry. The ratio of these two quantities is therefore fairly well defined, and is largely independent of modelbased assumptions. Thus one can estimate the $h_{3}$ amplitude that corresponds to the 45 $\mathrm{m} \mathrm{s}^{-1}$ curvature amplitude reported by GH. The resulting amplitude is $2.54 \%$, which is inconsistent with the measured $h_{3}$ amplitude with $10 \sigma$ significance. By this measure, our observations are therefore strongly inconsistent with those by $\mathrm{GH}$. On the other hand, if one assumes a nonradial pulsation with $\ell=5$ and an observed radial velocity amplitude of $56 \mathrm{~m}$ $\mathrm{s}^{-1}$, then our modeling shows that under typical circumstances the expected amplitudes of all $h_{i}$ coefficients should be below $0.5 \%$. Such amplitudes are only marginally detectable in our data; therefore, nonradial pulsations cannot be excluded by this test as the cause of the radial velocity signal in $51 \mathrm{Peg}$. The surface flow velocities associated with this pulsation would peak at about $5 \mathrm{~km} \mathrm{~s}^{-1}$, so the linearity assumption involved in scaling our model results would probably be violated. The dissipation associated with such near-sonic flows would likely cause larger line profile variations than those we calculated, however, so the actual limit on pulsation amplitude is probably smaller than that given above.

\section{B. $\tau$ Boötis}

The star $\tau$ Boo rotates more rapidly $\left(v \sin i=15 \mathrm{~km} \mathrm{~s}^{-1}\right)$, than does 51 Peg and the amplitude of its sinusoidal radial velocity variation $\left(468 \mathrm{~m} \mathrm{~s}^{-1}\right)$ is a factor of 9 larger (Marcy et al. 1997). Both of these circumstances make pulsations easier to detect in $\tau$ Boo than in $51 \mathrm{Peg}$. If the radial velocity variability of $\tau$ Boo were to arise from nonradial pulsations, then our models show that the anticipated signals in $h_{3}, h_{4}$, and $h_{5}$ should all have magnitudes of several percent.

We searched for such periodic signals in the line shapes of $\tau$ Boo just as we did in those of $51 \mathrm{Peg}$, by examining the periodograms and by fitting sinusoids to the known radial velocity period of $3.313 \mathrm{~d}$. The fitted values and their errors may be found in the last column of Table 1. Again, none of the fitted sinusoid amplitudes are significantly larger than the estimated errors, so no line shape changes at the radial velocity period have been detected. Since $\tau$ Boo is hotter than $51 \mathrm{Peg}$, its $\lambda 6252 \mathrm{~V}$ I line is too weak to allow a meaningful depth measurement. For this reason, Table 1 gives no line depth ratio estimate for this star.

If nonradial pulsations were responsible for $\tau$ Boo's radial velocity variation, our models lead us to expect an $h_{3}$ amplitude of about 15\%. Figure 2 shows the observed $h_{3}$ values for $\tau$ Boo phased to the radial velocity period, as well as a comparison between the fitted sinusoid and that expected from the pulsation models. In this case the results are unambiguous: pulsations with large enough amplitude to cause the radial velocity signal would also cause observable distortions of the line shapes, which are not seen.

The $h_{3}$ periodogram for $\tau$ Boo shows a wide region of excess power between $0.2 \mathrm{c} \mathrm{d}^{-1}$ and $0.4 \mathrm{c} \mathrm{d}^{-1}$. Its amplitude is small, however, (the rms signal associated with this entire band is about $0.6 \%$ ) and the bandwidth is comparable to the estimated rotation frequency of the star, which is thought to be identical to the radial velocity frequency. Perhaps this $h_{3}$ variation results from magnetic active regions rotating across the disk; it may also be associated with the excess radial velocity noise noted in $\tau$ Boo by Butler et al. (1997). In 
any case, it is too small in amplitude and covers too wide a frequency band to be identified directly with the radial velocity signal.

\section{Discussion}

To summarize our conclusions: (a) We find no evidence for pulsations in 51 Peg. Our upper limits on line shape variations are in significant conflict with the bisector curvature measurements by GH. (b) For the observed radial velocity signal, our models imply line shape variations that are too small to be readily detected, either by our methods or in GH's bisector data. Thus, pulsations are not excluded as an explanation for 51 Peg's radial velocity variability. (c) The radial velocity variability of $\tau$ Boo, whose period is similar to that of $51 \mathrm{Peg}$, does not arise from pulsations.

These conclusions have implications for the study of both extra-solar planets and of stellar pulsations. The absence of a pulsation signal in $\tau$ Boo supports the idea that companions of roughly jovian mass may exist in close orbits around Sun-like stars. If one such object circles $\tau$ Boo then it is plausible that another circles $51 \mathrm{Peg}$, questions about the companions' process of origin notwithstanding. As an issue in stellar seismology, the absence of large-amplitude (by solar standards) pulsations in Sun-like stars would be disappointing but not surprising. Our evidence regarding $51 \mathrm{Peg}$ itself is not conclusive, while the observations by GH raise interesting questions but lack confirmation. Further data on this star may therefore be desirable.

We are grateful to the rest of the AFOE team (Martin Krockenberger and Adam Contos) and to the staff at SAO's Whipple Observatory (Bastian van't Saant, Ted Groner, Perry Berlind, Jim Peters, and Wayne Peters) for their assistance in obtaining and reducing the observations described here. We thank Coen Schrijvers for providing test cases against which to compare our line profile simulation code, and Artie Hatzes for many useful discussions.

\section{References}

Abramowitz, M. \& Stegun, I. 1972, Handbook of Mathematical Functions, Dover, New York, p. 775

Brown, T.M., Noyes, R.W., Nisenson, P., Korzennik, S., \& Horner, S. 1994, PASP 106, 1285

Brown, T.M., Kotak, R., Horner, S.D., Kennelly, E.J., Korzennik, S., Nisenson, P. \& Noyes, R.W. 1998, ApJ Supp (submitted)

Butler, R.P., Marcy, G.W., Williams, E., Hauser, H., \& Shirts, P. 1997, ApJ Lett. 474, L115

Gray, D.F. 1997, Nature, 385, 795

Gray, D.F. \& Hatzes, A.P. 1997, ApJ (in press)

Hatzes, A.P. 1996, PASP 108, 839

Kennelly, E.J., Brown, T.M., Kotak, R., Sigut, T.A.A., Horner, S.D., Korzennik, S.G., Nisenson, P., Noyes, R.W., Walker, A., \& Yang, S. 1997, ApJ (in press) 
Marcy, G.W., Butler, R.P., Williams, E., Bildsten, L., Graham, J.R., Ghez, A.M., \& Jernigan, J.G. 1997, ApJ 481, 926

Mayor, M. \& Queloz, D. 1995, Nature, 378, 355

Noyes, R.W., Jha, S., Korzennik, S., Krockenberger, M., Nisenson, P., Brown, T.M., Kennelly, E.J., \& Horner, S.D. 1997, ApJ Lett. 483, 111

Schrijvers, C., Telting, J.H., Aerts, C., Ruymaekers, E., \& Henrichs, H.F. 1997, A\&A Supp. 121, 343

Vogt, SS. \& Penrod, G.D. 1983, ApJ, 275, 661 


\section{Table 1}

Observed Amplitudes and Errors for 51 Peg \& $\tau$ Boo

\begin{tabular}{clll}
\hline & $51 \mathrm{Peg}$ & $51 \mathrm{Peg}$ & $\tau$ Boo \\
& $4.231 \mathrm{~d}$ & $2.575 \mathrm{~d}$ & $3.313 \mathrm{~d}$ \\
& & & \\
\hline$h_{3}(\%)$ & $0.147 \pm 0.26$ & $0.344 \pm 0.38$ & $0.270 \pm 0.35$ \\
$h_{4}(\%)$ & $0.272 \pm 0.42$ & $0.238 \pm 0.45$ & $0.148 \pm 0.30$ \\
$h_{5}(\%)$ & $0.219 \pm 0.19$ & $0.323 \pm 0.19$ & $0.190 \pm 0.17$ \\
Line Ratio & $0.277 \pm 0.30$ & $0.416 \pm 0.28$ & - \\
\hline
\end{tabular}




\section{Figure Captions}

Figure 1. The line shape parameter $h_{3}$ for 51 Peg plotted against phase of the radial velocity variation. Filled circles are data points covering one cycle of the RV variation; open circles are the same data covering a wider phase range, plotted for clarity of the phase relations. The solid curve is a least-squares sine wave fit to the observations, with amplitude $0.147 \%$. The other curves are the typical $h_{3}$ signals expected from pulsation models yielding (dashed) an RV signal $V_{d o p}=56 \mathrm{~m} \mathrm{~s}^{-1}$, and (dot-dashed) a line bisector curvature signal of $45 \mathrm{~m} \mathrm{~s}^{-1}$.

Figure 2. Same as Fig. 1, but for $\tau$ Boo. In this case no bisector curvature information is available, so the corresponding (dot-dashed) curve is not shown. 
51 Peg Avg. h3

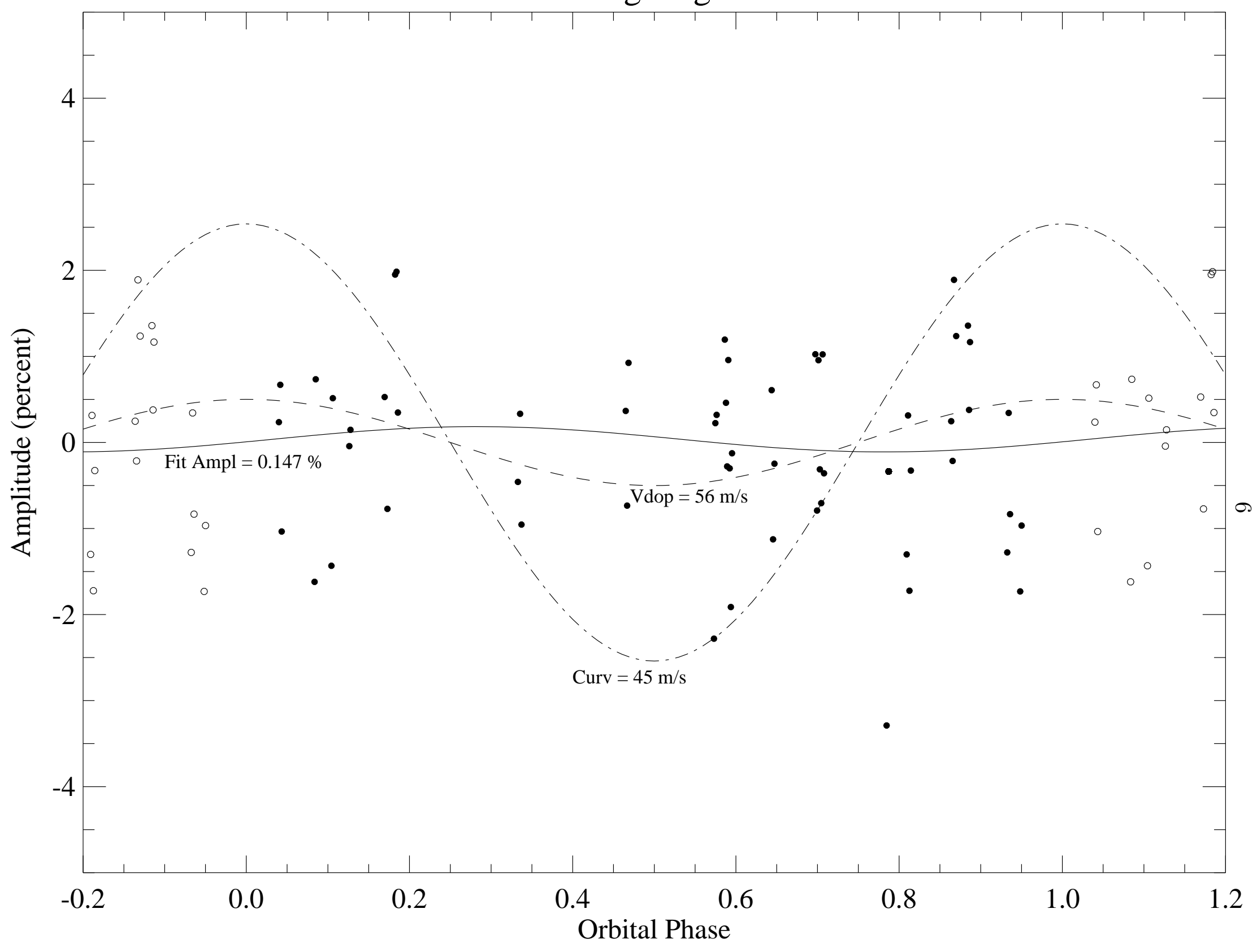


tau Boo Avg. h3

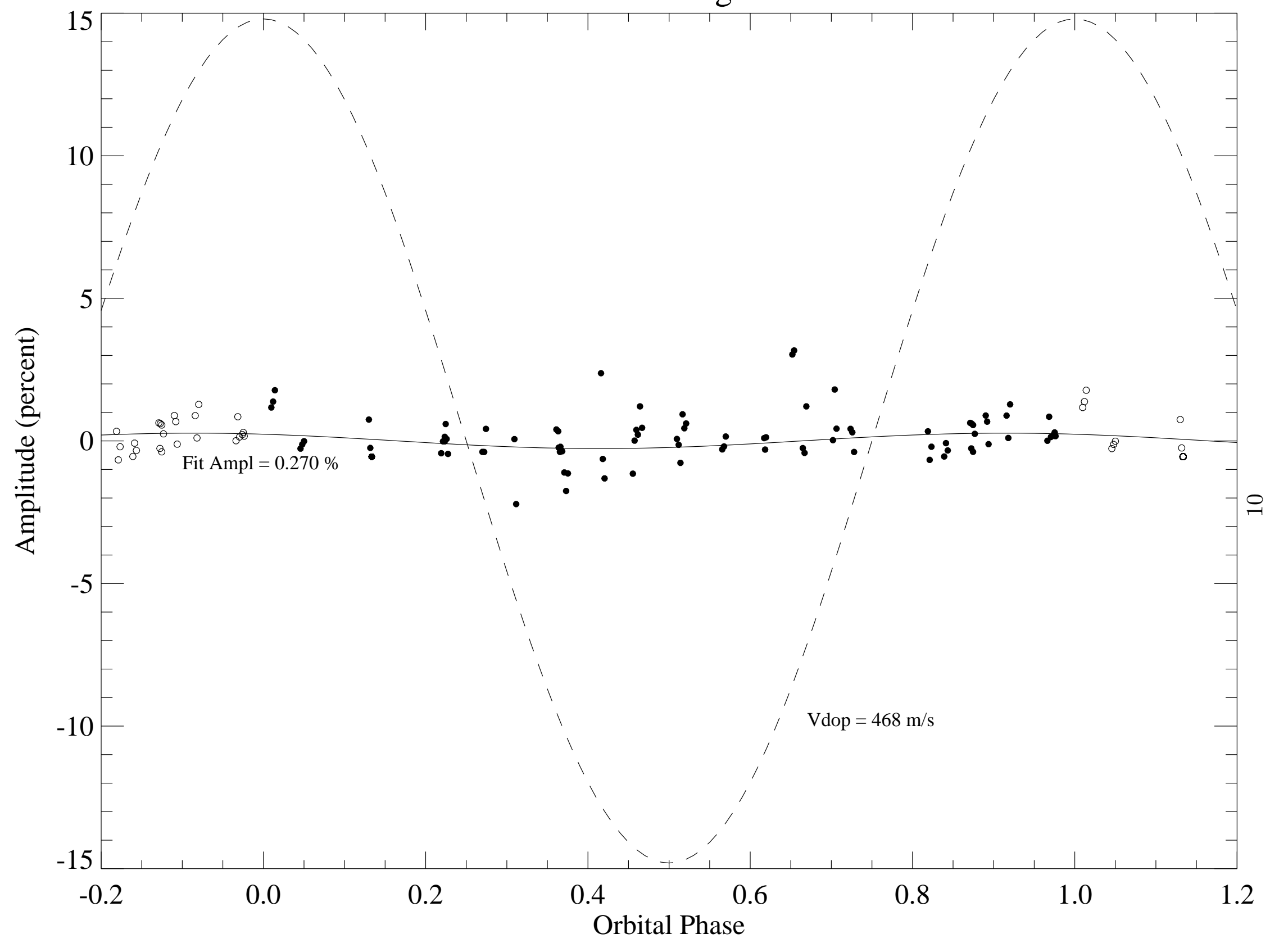

